\title{
Bioinformatics Analysis of ISLR in Pan-Cancer and its Correlation with Tumor Immunity
}

\section{Cangyuan Zhang}

The Yangzhou School of Clinical Medicine of Dalian Medical University

\section{Ziyang Long}

The Yangzhou School of Clinical Medicine of Dalian Medical University

\section{Cheng Yan}

The Yangzhou School of Clinical Medicine of Dalian Medical University

\section{Mohamed Said Jalloh}

Clinical Medical College, Yangzhou University

\section{Yongkun Fang}

The Yangzhou School of Clinical Medicine of Dalian Medical University

\section{Li Bao}

Clinical Medical College, Yangzhou University

\section{Biao Sun}

Clinical Medical College, Yangzhou University

\section{Wenzhe Shao}

The Yangzhou School of Clinical Medicine of Dalian Medical University

\section{Guowei Sun}

The Yangzhou School of Clinical Medicine of Dalian Medical University

\section{Yayan Fu}

Clinical Medical College, Yangzhou University

\section{Qi Zhang}

Clinical Medical College, Yangzhou University

\section{Wei Wang}

The Yangzhou School of Clinical Medicine of Dalian Medical University

\section{Liuhua Wang}

General Surgery Institute of Yangzhou, Department of Gastrointestinal Surgery, Northern Jiangsu People's Hospital Affiliated to Yangzhou University

\section{Jun Ren}

General Surgery Institute of Yangzhou, Department of Gastrointestinal Surgery, Northern Jiangsu People's Hospital Affiliated to Yangzhou University

\section{Qiannan Sun}

General Surgery Institute of Yangzhou, Department of Gastrointestinal Surgery, Northern Jiangsu People's Hospital Affiliated to Yangzhou University 


\section{Dong Tang}

General Surgery Institute of Yangzhou, Department of Gastrointestinal Surgery, Northern Jiangsu People's Hospital Affiliated to Yangzhou University

\section{Daorong Wang ( $\sim$ wdaorong666@sina.com)}

General Surgery Institute of Yangzhou, Department of Gastrointestinal Surgery, Northern Jiangsu People's Hospital Affiliated to Yangzhou University

\section{Research Article}

Keywords: ISLR, pan-cancer, bioinformatics analysis, prognosis, immune infiltration, tumor microenvironment

Posted Date: October 28th, 2021

DOI: https://doi.org/10.21203/rs.3.rs-970091/v1

License: (c) (i) This work is licensed under a Creative Commons Attribution 4.0 International License. Read Full License 


\section{Abstract \\ Background}

The protein meflin encoded by ISLR contains a C2-type immunoglobulin (Ig)-like domain and five leucinerich repeat (LRR) domains. ISLR is known to play a role in a small number of tumors, but its role in most tumors is unknown. The purpose of this study was to analyze the expression and prognosis of ISLR in pan-cancer, as well as its correlation with tumor immunity.

\section{Methods}

We used multiple databases and $\mathrm{R}$ software to conduct bioinformatics analysis to explore the predictive role of ISLR in pan-cancer, mainly involving expression patterns, prognosis, and immune infiltration.

\section{Results}

Compared with normal tissues, the expression of ISLR was significantly increased or decreased in most tumors. Moreover, the high expression of ISLR may cause the prognosis of some tumors to become better or worse. ISLR also affects immune infiltration in a variety of tumors, which affects the clinical prognosis. ISLR is also significantly related to TMB and MSI in pan-cancer and is related to genes encoding immune regulatory genes. ISLR also affects various cancer-and immune-related pathways.

\section{Conclusions}

ISLR is differentially expressed in tumors, may regulate TME, affect tumor prognosis, and is expected to become a prognostic biomarker.

\section{Introduction}

Despite the combined application of surgical therapy, radiotherapy, chemotherapy, and targeted therapy, cancer mortality and recurrence rates have been significantly reduced. However, cancer is still one of the biggest health problems in the world and the second leading cause of death in the United States[1]. One of the reasons for the current dilemma is that the molecular mechanisms of cancer are not fully understood. Differential expression and abnormal gene mutations affect the occurrence and progression of tumors. It is particularly important to explore the molecular mechanism of cancer to enhance the effect of chemotherapy and targeted therapy to improve patient survival rates and reduce tumor recurrence rates.

ISLR (immunoglobulin superfamily containing leucine rich repeat), located at 15q24.1, which encodes a conserved protein (Meflin) consisting of a C2-type immunoglobulin (Ig) -like domain and five leucine-rich 
repeat (LRR) domains[2]. These rich domains of ISLR can influence protein-protein interactions or cell adhesion. ISLR also regulates the undifferentiated state of cells and is a potential marker of mesenchymal stromal cells[3]. ISLR can also promote regeneration and repair of heart tissues[4, 5]. In addition, ISLR plays an important role in intestinal regeneration and tumorigenesis by influencing the Hippo-YAP signaling pathway in epithelial cells[6]. ISLR in CAFs also plays a role in inhibiting pancreatic cancer[7]. At present, only a few studies have found that ISLR may play a role in certain cancers, but the role of ISLR in pan-cancer is unclear.

Recently, the tumor microenvironment (TME) has been found to play an important role in tumor development $[8,9]$. The TME consists mainly of stromal and cellular components. Cell components mainly include immune cells, stromal cells, endothelial cells, and cancer-associated fibroblasts (CAFs). Among these, immune cells account for an important proportion of cells [10]. With the advent of immunotherapy, an increasing number of studies on tumor-related immunity are being conducted. The traditional view of immune cells that inhibit tumor progression is challenging. In recent years, it has been suggested that immune cell infiltration in the TME may provide more convenience for tumor escape[11, 12]. In addition, CAFs can produce extracellular matrix (ECM), secreting a variety of cytokines and other substances to promote tumor growth and invasion, but can also play a role in inhibiting tumor progression in some cancers $[13,14]$. Therefore, exploring the role of ISLR in the TME is helpful in providing a deeper understanding of tumor progression.

Here, we used multiple databases such as Oncomine, TGCA, GEO, and TIMER to analyze the role of ISLR in tumors. The results demonstrate that ISLR is related to the development of some tumors and is expected to be a good marker for diagnosis, treatment, and prognosis. This article lays a foundation for exploring the mechanism of ISLR in various cancers and is expected to guide further research on ISLR in cancer.

\section{Methods}

\subsection{ISLR expression data downloaded in human tumor and normal tissues}

Oncomine (https://www.oncomine.org/) is an online cancer microarray database that contains a large amount of gene expression data points from a variety of cancer types[15]. We analyzed the ISLR expression of pan-cancer in the Oncomine database. Enter the gene symbol "ISLR" and set the data type to "mRNA" The thresholds included multiple variations: 2 , P value: 0.001 , and gene grade: $10 \%$.

We downloaded normal tissue data and 33 tumor-related (TCGA-FPKM-processed) RNA sequencing datasets as well as phenotypic and survival data from the UCSC Xena website (https://xena.ucsc.edu/, derived from The Cancer Genome Atlas (TCGA) and Genotype-Tissue Expression (GTEx)[16]. We then used Perl software and R software to extract the ISLR expression data from the data and conducted extensive cancer analysis. Some of the data were analyzed using sangerbox online tools 

$19131, G=60499)$. The results are shown in the box diagram.

\subsection{Analysis of the correlation between ISLR expression levels and clinical characteristics of pan-cancer and survival analysis in pan-cancer}

The Kaplan-Meier Plotter (https://kmplot.com/analysis/) is a powerful online tool for assessing the impact of survival in 21 cancer types[17]. The Kaplan-Meier plotter was used to analyze the relationship between ISLR expression and overall survival (OS) and relapse-free survival (RFS) in tumors. R software (version 4.1.1, www.r-project.org) and the Kaplan-Meier survival " package were used to search for ISLR expression levels in TCGA datasets and group them according to median values to determine their relationship with prognosis, including overall survival (OS), progression-free survival (PFS), disease-free survival (DFS), and disease-specific survival (DSS). "Survminer" and "survival" packages are used to draw survival curves. The results of Cox regression were visualized using the "forestplot" package. The $R$ package "ggpubr" is used to analyze the relationship between ISLR and clinical features.

\subsection{Correlation between ISLR expression and tumor immune microenvironment invasion}

The relationship between ISLR expression and immune infiltration was analyzed using TIMER (http://cistrome.org/TIMER/). TIMER is a method that can systematically analyze the abundance of immune infiltration in various cancer types[18]. The TIMER database contains samples from the TCGA database for 32 cancer types. We analyzed whether ISLR expression is related to immune-infiltrating cells. We calculated the median value of ISLR expression and classified data greater than the median value into the high expression group, and data below the median value into the low expression group. We then used KM analysis to observe the relationship between immune cells and prognosis. We use the Surv (CancerType) variable simultaneously to customize the formula of the Cox regression model. This model is composed of the function coxph () from the R package 'survival'. HR gives the hazard ratio, and its upper and lower $95 \%$ confidence intervals are shown in $95 \% \mathrm{Cl} \_\mathrm{I}$ and $95 \% \mathrm{Cl} \_$u. We also analyzed whether ISLR expression is related to tumor purity.

\subsection{ISLR expression is associated with tumor mutation load (TMB), microsatellite instability (MSI), immune gene modulators, and immunotherapy in cancers}

We used the Tne Perl script to obtain TMB data from the TCGA database. TCGA was used to extract MSI scores. The correlation between ISLR expression and TMB or MSI was analyzed using R software. We generated a visual radar map using the R package "FMSB." We also used the TISIDB 
(http://cis.hku.hk/TISIDB/) online tool to analyze the correlation between ISLR expression and immune activators, inhibitors, and major histocompatibility complex) molecules[19]. Subsequently, TISIDB was used to visualize the influence of ISLR expression on the effect of tumor immunotherapy.

\subsection{Gene Set Enrichment Analysis (GSEA) analysis of ISLR in tumors}

We use R packages "clusterProfiler"[20], "enrichPlot, "', and org.hs.eg. db" and "limma" to perform ISLR GSEA (https://www.gsea-msigdb.org/gsea/downloads.jsp) analysis in tumors, Including KEGG (Kyoto Encyclopedia of Genes and Genomes) and GO (Gene Ontology).

\subsection{Statistical analysis}

All TCGA-FPKM gene expression data were normalized using Log2 $(x+1)$ transformation. A t-test was used to assess the differences between the normal and tumor tissues. Kaplan-Meier and Cox proportional risk model methods were used to assess patient prognosis. We used the log-rank test for the survival curve for data sorting and data display. The correlation of gene expression was assessed using the Spearman test or Pearson's test. If there is no special note, we consider that $P<0.05$, is considered statistically different. Statistical analysis was performed using R software (version 4.1.1).

\section{Results}

\subsection{The expression of ISLR in various normal tissues}

ISLR mRNA expression was extracted from the TOIL RSEM FPKM data from GTEx. The expression amount of ISLR in normal tissues of each organ was expressed by "ggpubr" package of R software, and the box diagram was drawn. The results indicated that there were differences between genders in muscle, heart, breast, adrenal gland, skin, and nerve tissues $(P<0.05)$, but there was no statistical difference between genders in other organs (Figure 1B).

\subsection{Differential expression of ISLR in pan-cancer and its correlation with tumor stage}

We used the Oncomine database to analyze ISLR mRNA expression levels in pan-cancer. The results showed that there were more data sets of high ISLR expression in several cancer groups, including colorectal, esophageal, gastric, liver, and pancreatic cancers, as well as leukemia, lymphoma, and sarcoma, compared with normal groups. At the same time, there were many datasets with low ISLR expression in bladder cancer, breast cancer, kidney cancer, and ovarian cancer (Figure 1A). Supplementary Table1a provides details of ISLR expression in each tumor.

To further evaluate ISLR expression in generalized carcinoma, RNA sequencing data in TCGA-FPKM were analyzed by $\mathrm{R}$ software using Log2 $(\mathrm{x}+1)$ transformation. ISLR was differentially expressed in tumors and adjacent normal tissues (Figure 1C). ISLR expression is significantly downregulated in bladder urothelial 
carcinoma (BLCA), breast invasive carcinoma (BRCA), cervical squamous cell carcinoma and endocervical adenocarcinoma (CESC), kidney chromophobe (KICH), kidney renal clear cell carcinoma (KIRC), kidney renal papillary cell carcinoma (KIRP), liver hepatocellular carcinoma (LIHC), prostate adenocarcinoma (PRAD), thyroid carcinoma, THCA and uterine corpus endometrial carcinoma (UCEC). In contrast, ISLR expression was higher in colon adenocarcinoma (COAD), cholangiocarcinoma (CHOL), lung adenocarcinoma (LUAD), head and neck squamous cell carcinoma (HNSC), and esophageal carcinoma (ESCA) than in the adjacent normal tissues.

Considering the small amount of normal tissue expression in TCGA, we used the Sangerbox website to analyze the unified standardized universal cancer dataset downloaded from the UCSC database: TCGA TARGET GTEx (PANCAN,N=19131,G=60499). Furthermore, the expression data of the ISLR gene in each sample were extracted, and log2 $(x+1)$ transformation was performed for each expression value to exclude cancer species with less than three samples in a single cancer species. Finally, the expression data of 34 cancer species were obtained. The results showed that ISLR was differentially expressed in tumors other than rectal adenocarcinoma (READ), testicular germ cell tumors (TGCT), LUAD, BRCA, pheochromocytoma, and paraganglioma (PCPG) (Figure 1E). See Supplementary Table1b and Supplementary Table1c.

Next, we used R software to analyze the correlation between ISLR expression and tumor stage based on the clinical data of tumor patients downloaded from TCGA. The expression of ISLR was significantly different between the BLCA, BRCA, COAD, ESCA, KIRC, KIRP, STAD, and TGCT $(P<0.05)$. See Figure1D. All supplementary materials are summarized in Figure 1.

\subsection{Prognostic value of ISLR in pan-cancer}

We used R software and online database websites to analyze the prognostic significance of ISLR in pancancer. We used the Kaplan-Meier Plotter online tool to find the best cutoff value for ISLR expression and classify the data higher than the best cutoff value into the high expression group, and the remaining data into the low expression group. We found that differential expression of ISLR affects overall survival (OS) prognosis. The results showed that the high expression of ISLR was significantly related to the poor prognosis of BLCA, STAD, ovarian cancer (OV), KIRC, and KIRP. Meanwhile, the low expression of ISLR was significantly related to the poor overall prognosis of CESC, UCEC, HNSC, and other tumors (Figure 2). All P-values were $<0.05$. COX analysis of TCGA data using R package "survival" showed that the high expression of ISLR in 7 tumors (KIRP, BLCA, LGG (brain lower grade glioma), KIRC, STAD, ACC (adrenocortical carcinoma), and PRAD) leads to a poor overall prognosis. COX survival analysis data were obtained from UCSC. See Supplementary Figure 2 for further details.

We then analyzed the significance of ISLR expression in relapse-free survival (RFS). High expression of ISLR may cause short RFS in GC, OV, pancreatic adenocarcinoma (PAAD), LUAD, esophageal adenocarcinoma, esophageal squamous cell carcinoma, TGCT, and HNSC. Low expression of ISLR is associated with short RFS in hepatocellular carcinoma, thyroid cancer, endometrial cancer, and other cancers (Figure 3). 
Next, according to the expression data of cancer species and the corresponding survival data obtained from UCSC, we used R software to explore the correlation between ISLR expression and disease-free survival (DFS), progression-free survival (PFS), and disease-free survival (DSS). COX analysis is based on the coxph function of $\mathrm{R}$ software and the application of the log-rank test for statistical testing to obtain prognostic significance. In addition, we divided patients into two groups with high and low expression according to the median value of ISLR expression, and assessed the impact of ISLR on prognosis through $\mathrm{KM}$ analysis. For DFS, COX analysis and KM analysis both show that high ISLR expression closely affects the poor prognosis of tumors such as ACC, ESCA, and PAAD. In contrast, low ISLR expression affects the poor prognosis of tumors such as THCA and UCEC. For PFS, COX analysis and KM analysis showed that high expression of ISLR was only closely related to the poor prognosis of KIRC. For DSS, COX analysis and KM analysis both show that the high expression of ISLR is closely related to the poor prognosis of tumors such as STAD, KIRC and KIRP (Figure 4). See Supplementary Information for details in Supplementary Figure DFS, Supplementary Figure DSS and Supplementary Figure PFS.

\subsection{Correlation between ISLR expression and tumor microenvironment scores}

Tumor microenvironment scores were obtained through tumor microenvironment analysis, including stromal and immune scores, and ESTIMATE scores. Then, correlation analysis between ISLR expression and tumor microenvironment scores was carried out. The results showed that in 16 tumor types (BLCA, CHOL, COAD, READ, GBM (glioblastoma multiforme), KICH, KIRP, LIHC, LUAD, LUSC (lung squamous cell carcinoma), PAAD, PRAD, SKCM (skin cutaneous melanoma), STAD, THYM (thymoma), PCPG), the ISLR gene may have a significant correlation with tumor stromal and immune scores. The expression of ISLR in most of the remaining cancers was mostly correlated with the stromal scores, and there was no obvious correlation with the immune scores. Part of this is shown in Figure 5.

\subsection{Analysis of the correlation between ISLR expression and immune cell content and the relationship between immune cells and prognosis}

To further study the regulatory mechanism of ISLR in TME immunity, we used TIMER to examine the relationship between ISLR and immune cell content. Combining COX and KM analysis results showed that in 11 types of tumors, ISLR expression was negatively correlated with tumor purity and was highly correlated with immune cell content. Infiltration of immune cells affects the prognosis of patients. ISLR expression was positively correlated with the amount of CD8+ T cell infiltration in the BLCA (Figure 6A) and KIRP (Figure $6 \mathrm{~K}$ ), and the prognosis of patients with tumors with high CD8+ T cell infiltration is poor. The high expression of ISLR causes high CD4+ T cell infiltration in CESCs, while a high amount of CD4+ T cell infiltration leads to a better prognosis (Figure 6B). In addition, the prognosis of patients with high $\mathrm{B}$ cell infiltration in KIRP was poor, and it was positively correlated with ISLR expression (Figure 6K). This means that the high expression of ISLR may promote tumor progression and reduce the survival rate of 
patients by upregulating B cells and CD8+ T cells in KIRP. The data are the same as the OS and DSS of $\mathrm{KIRP}$ in Figures 2 and 4. In contrast, although the high expression of ISLR caused high $\mathrm{B}$ cell infiltration of HNSC (Figure 6D) and LUAD (Figure 6F), the low amount of B cell infiltration leads to a poor prognosis. This is consistent with the poor prognosis of OS caused by the high expression of ISLR in HNSC. In addition, ISLR expression was directly proportional to dendritic cells in GBM (Figure 6C), OV (Figure 6H), and SKCM (Figure 6I). The difference is that the prognosis of dendritic cells is poor when the amount of infiltration is high in GBM, and the prognosis is poor when the amount of infiltration is low in OV and SKCM. The OV results were contrary to the above-mentioned prognostic results. In addition to immune infiltration, other factors may also affect patient prognosis. The high infiltration of macrophages in LGG (Figure 6E) and STAD (Figure 6J) results in a poor prognosis for patients. However, the amount of macrophage infiltration is inversely proportional to the expression of ISLR in LGG; on the contrary, it is proportional to the expression of ISLR in STAD. Combined with the OS results of ISLR in STAD, it can be predicted that ISLR may increase the amount of macrophage infiltration in the TME to promote tumor development and affect prognosis. The lower the neutrophil infiltration in MESO (Figure 6G) and UVM (Figure 6L), the worse the prognosis. Neutrophils are negatively correlated with ISLR expression in MESO and positively correlated with ISLR in UVM. The correlation between the content of immune cells in the remaining tumors and the Cox regression data are shown in the appendix.

We next examined whether ISLR expression affects the infiltration of immune cell subtypes. We set the filter conditions as $P<0.001$ and cor $<0.3$. The results of the study showed that in BLCA, ESCA, GBM, KICH, KIRP, LGG, PRAD, STAD, TGCT, THYM cancer types, ISLR expression, and the level of immune cell infiltration were significantly related (see Figure 7). The high expression of ISLR in BLCA, ESCA, STAD, TGCT, and THYM leads to high infiltration of resting mast cells. ISLR expression is proportional to the content of Macrophages M0 in GBM and LGG, and proportional to Macrophages M1 in LGG, and proportional to Macrophages M2 in TGCT. ISLR was negatively correlated with the monocyte count in the LGG. It is not difficult to find that the high expression of ISLR may play a role in promoting the transformation of monocytes to macrophages in LGG. ISLR expression and activated NK cell infiltration were positively correlated with $\mathrm{KICH}$. In addition, ISLR is inversely proportional to naive B cells in GBM, TGCT, and THYM. ISLR and plasma cells were positively correlated in KIRP but negatively correlated in PRAD and THYM. High expression of ISLR leads to high infiltration of CD8 + T cells in $\mathrm{KICH}$ and LGG. At the same time, ISLR expression in TGCT and THYM was negatively correlated with the infiltration of activated memory CD4 T cells. In addition, in BLCA and TGCT, ISLR expression was inversely proportional to follicular T cells (Figure 7). See the Supplementary Materials in Figure 7 for details.

\subsection{ISLR expression affects TMB, MSI and immunomodulatory gene in pan-cancer}

Next, we used the TISIDB online tool to obtain ISLR expression and immune-related heat maps. We selected each gene whose heat was related to ISLR expression and drew a scatter diagram, as shown in Figure below (Figure 8A-C). TMEM173 had the greatest positive correlation with ISLR, and IL6R had the greatest negative correlation with ISLR. HLA-DPB1 expression was significantly and positively correlated. 
We also studied whether ISLR expression affected the TMB and MSI. TMB and MSI are closely related to cancer immune checkpoints[21,22]. The results indicated that ISLR expression was associated with TMB in 20 cancer types (Figure 8D). On the one hand, ISLR is positively correlated with the TMB of acute myeloid leukemia (LAML), LGG, OV, and THYM. In contrast, ISLR negatively correlated with TMB in CHOL, GBM, HNSC, KIRC, KIRP, LIHC, LUAD, LUSC, PCPG, PRAD, READ, SKCM, STAD, THCA, UCEC, and uterine carcinosarcoma (UCS). We further found that ISLR expression was negatively correlated with MSI in nine cancer types, including CHOL, HNSC, KIRC, LUSC, PAAD, PCPG, SKCM, STAD, and UCEC (Figure 8E). Next, we used the TISIDB online tool to analyze the relationship between ISLR expression in tumors and the effect of immunotherapy (Figure 8F). The results showed that in melanoma, the high expression of ISLR had a significant impact on the poor immunotherapy effect $(P<0.05)$.

\subsection{Correlation between ISLR expression and multiple cancer-related pathways}

Next, we analyzed GO function annotations and KEGG pathways related to ISLR in various cancers (below). The data show that the high expression group of ISLR is enriched in some cancer-related pathways, including the MAPK, TGF_BETA, JAK, and some cytokine and metabolic pathways (Figure 9A F). GO analysis of ISLR involves the activation of certain immune cells or immune pathways, as well as the conduction of certain signaling pathways. Part of the analysis is shown in the figure below (Figure $9 \mathrm{G}$ $-\mathrm{L})$.

\section{Discussion}

Although ISLR was discovered as early as 1997, it has not been fully studied in tumors. Some studies have reported that ISLR plays an important role in tumor progression in CAFs, such as colorectal cancer, pancreatic cancer, and malignant pleural stromal tumors $[7,23,24]$. This means that ISLR may play a role in the TME, which is one of the reasons why we conducted this research on the role of ISLR in pan-cancer and tumor immunity.

Based on the combined results of TCGA and GTEx data, we found that the ISLR gene was highly expressed in 11 tumors (GBM, GBMLGG (glioma), LGG, STES (stomach and esophageal carcinoma), STAD, HNSC, WT (high-risk wilms tumor), THCA, PAAD, LAML, CHOL) and is expressed at low levels in 18 cancers (UCEC, CESC, ESCA, KIRP, KIPAN (pan-kidney cohort), COAD, COADREAD, PRAD, KIRC, LUSC, LIHC, SKCM, BLCA, OV, UCS, ALL (acute lymphoblastic leukemia), ACC, KICH). In addition, we found that ISLR expression was correlated with the overall staging of tumors such as BLCA, BRCA, COAD, ESCA, KIRC, KIRP, STAD, and TGCT. The results showed that the expression of ISLR had a greater relationship with the grades of gastrointestinal tumors and urinary system tumors. We then analyzed the relationship between ISLR expression and patient prognosis and found that the high expression of ISLR in STAD, OV, KIRC, KIRP, and BLCA would lead to poor OS. In contrast, the low expression of ISLR in UCEC, HNSC, and CESC caused the OS of the patient to be poor. The prognostic results of ISLR in STAD, UCSC, and CESC were consistent with the results of expression differences. The inconsistency between the remaining tumor 
prognosis results and the differential expression results may be related to other biological factors, such as epigenetics or limited sample data. The specific reasons for this are still awaiting further study. In addition, we found that ISLR also affects the prognosis of RFS, DFS, DSS, and PFS in pan-cancer. The results indicated that the high expression of ISLR not only caused the poor prognosis of STAD patients with OS, but also caused the poor prognosis of STAD patients with RFS and DSS. Similarly, high expression of ISLR can lead to poor prognosis of OV patients with RFS, poor prognosis of KIRP patients with DSS, and poor prognosis of KIRC patients with DSS and PFS. In addition, the low expression of ISLR not only affects the poor prognosis of UCEC patients with OS, but also leads to poor prognosis in patients with RFS and DFS. Although the high expression of ISLR leads to a better prognosis for OS in patients with HNSC, it leads to a poor prognosis for RFS. This may be due to its small size. Although ISLR expression was not correlated with OS prognosis in ESCA, PAAD, and THCA patients, it affected the prognosis of RFS and DFS in PAAD and THCA patients, and also had a significant correlation with RFS and DSS in ESCA patients. These results indicate that the ISLR is expected to become a potential prognostic marker for a variety of cancers.

The TME plays a key role in regulating tumor progression and regulating immunotherapy[25, 26]. Therapies targeting TME, including immunotherapy, anti-angiogenic drugs, and treatments for cancerrelated fibroblasts and extracellular matrix, have great potential in the future[27]. We found that ISLR affected the tumor immunity. We used TCGA data for analysis and found that ISLR expression was directly proportional to the stromal scores of BLCA, CHOL, COAD, READ, GBM, KICH, KIRP, LIHC, LUAD, LUSC, PAAD, PRAD, SKCM, STAD, and THYM. ISLR expression in THYM is directly proportional to the stromal score and inversely proportional to the immune score. ISLR expression is directly proportional to ACC, BRCA, CESC, ESCA, HNSC, KIRC, LGG, MESO, OV, TGCT, UCEC, and UVM stromal scores. In addition, based on the relationship between immune cell infiltration and prognosis, combined with the correlation between ISLR expression and immune cell infiltration, we conclude that ISLR expression can affect the prognosis of patients by affecting the amount of immune cell infiltration. In BLCA, GBM, STAD, and KIRP, high expression of ISLR causes high infiltration of immune cells, and the high infiltration of immune cells will cause poor prognosis in patients. In CESC, HNSC, LUAD, OV, SKCM, UVM, high expression of ISLR causes high infiltration of immune cells, and the low infiltration of immune cells can cause poor prognosis. In LGG, low ISLR expression is directly proportional to high immune cell infiltration, and high immune cell infiltration can cause poor prognosis. In MESO, high expression of ISLR is directly proportional to low immune cell infiltration, and low immune cell infiltration will cause poor prognosis. Subsequently, we found that ISLR may affect the conversion of immune cell subtypes. To further verify the role of ISLR in immunity, we analyzed the role of ISLR in pan-cancer and immune genes and found that ISLR is significantly correlated with a variety of immune genes and immune checkpoints.

Some studies have shown that TMB is related to the clinical response of immune checkpoints to block immunotherapy in certain tumors. It is also believed that TMB can independently predict the response to anti-PD-1 therapy in a variety of tumors[28-30]. MSI refers to the mutation of mismatch repair (MMR) gene, which causes changes in DNA repetitive sequences (microsatellites) and affects tumor progression. MSI status can aggravate or alleviate the resistance of immune checkpoint inhibitors in patients by 
changing the TME in tumors[31]. Our results showed that ISLR has a certain correlation with TMB and MSI in some tumors. Combining the correlation between ISLR and immune genes, we hypothesized that the expression of ISLR had a significant impact on immunotherapy. This conclusion has been verified in patients with melanoma. Higher expression of ISLR leads to poor immunotherapy effects. Finally, our enrichment analysis proved that ISLR may affect the pathogenesis or prognosis of tumors by playing a role in a variety of common cancer pathways and immune-related pathways.

In conclusion, this ISLR pan-cancer analysis found that ISLR has high or low expression in most tumors compared to normal tissues and is different from multiple prognostic types. The impact of ISLR on each tumor is different, and its impact on prognosis may be related to TMB, MSI, and immune cell infiltration in some tumors. These results may help clarify the causes of tumor occurrence and development and provide new research ideas and directions for solving the drug resistance of tumor immunotherapy. However, the specific impact mechanism of ISLR on tumors is still waiting for us to further study.

\section{Abbreviations}

ACC Adrenocortical carcinoma

ALL Acute Lymphoblastic Leukemia

BLCA Bladder Urothelial Carcinoma

BRCA Breast invasive carcinoma

CAF Cancer-associated Fibroblast

CESC Cervical squamous cell carcinoma and endocervical adenocarcinoma

CHOL Cholangiocarcinoma

COAD Colon adenocarcinoma

COADREAD Colon adenocarcinoma/Rectum adenocarcinoma Esophageal carcinoma

DFS Disease-free Survival

DLBC Lymphoid Neoplasm Diffuse Large B-cell Lymphoma

DSS Disease-specific Survival

ECM Extracellular Matrix

ESCA Esophageal carcinoma

FPPP FFPE Pilot Phase II 
GBM Glioblastoma multiforme

GBMLGG Glioma

GEO Gene Expression Omnibus

GO Gene Ontology

GSEA Gene Set Enrichment Analysis

GTEx Genotype-tissue Expression

HNSC Head and Neck squamous cell carcinoma

Ig Immunoglobulin

ISLR Immunoglobulin Superfamily Containing Leucine Rich Repeat

KEGG Kyoto Encyclopedia of Genes and Genomes

$\mathrm{KICH}$ Kidney Chromophobe

KIPAN Pan-kidney cohort (KICH+KIRC+KIRP)

KIRC Kidney renal clear cell carcinoma

KIRP Kidney renal papillary cell carcinoma

LAML Acute Myeloid Leukemia

LGG Brain Lower Grade Glioma

LIHC Liver hepatocellular carcinoma

LRR Leucine-rich Repeat

LUAD Lung adenocarcinoma

LUSC Lung squamous cell carcinoma

MESO Mesothelioma

MHC Major Histocompatibility Complex

MSI Microsatellite Instability

OS Overall Survival 
OV Ovarian serous cystadenocarcinoma

PAAD Pancreatic adenocarcinoma

PCPG Pheochromocytoma and Paraganglioma

PFS Progression-free Survival

PRAD Prostate adenocarcinoma

READ Rectum adenocarcinoma

RFS Relapse-free Survival

SARC Sarcoma

SKCM Skin Cutaneous Melanoma

STAD Stomach adenocarcinoma

STES Stomach and Esophageal carcinoma

TGCA The Cancer Genome Atlas

TGCT Testicular Germ Cell Tumors

THCA Thyroid carcinoma

THYM Thymoma

TMB Tumor Mutation Load

TME Tumor Microenvironment

UCEC Uterine Corpus Endometrial Carcinoma

UCS Uterine Carcinosarcoma

UVM Uveal Melanoma

WT High-Risk Wilms Tumor

\section{Declarations}

Ethics approval and consent to participate 
Not applicable.

Consent for publication

Not applicable.

Availability of data and materials $\rrbracket$

The datasets analysed in this research were downloaded from TCGA (https://portal.gdc.cancer.gov/), TARGET (https://ocg.cancer.gov/programs/target), GTEx (https://gtexportal. org/home/), GEO (https://www.ncbi.nlm.nih.gov/geo/). Part of the data comes from Oncomine (https://www.oncomine.org/). Kaplan-Meier Plotter (https://kmplot.com/analysis/) is used to analyze the prognosis. Part of the immunization data comes from TIMER (http://cistrome.org/TIMER/). Part of the data generated or analyzed during this study is included in this published article and its supplementary information file.

Competing interests

The authors have no conflicts of interest to declare.

Funding:

This research was supported by the National Natural Science Foundation of China (81972269).

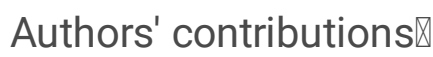

$\mathrm{CZ}, \mathrm{ZL}$, and $\mathrm{CY}$ designed the study and analyzed the data in this study. MSJ assisted with CZ for drafting the manuscript. YF, LB, BS, and WS conducted the data management. GS, YF, QZ, WW, and LW provided technical support and data correction. JR, QS, and DT-assisted DW in revising the manuscript. All authors reviewed the final version of the manuscript. CZ, ZL, and $\mathrm{CY}$ contributed equally to this study

Acknowledgements $\rrbracket$

The authors thank The Cancer Genome Atlas (TCGA), Genotype-Tissue Expression (GTEx), Gene Expression Omnibus (GEO), Target, Oncomine, TIMER, and Kaplan-Meier Plotter program staff . to provide open access to the database.

\section{Disclosure:}

TCGA, GEO, GTEx, Target, Oncomine, TIMER, and Kaplan-Meier Plotter belong to public databases. Patients involved in the database obtained ethical approval. Users can download relevant data free of research and publish relevant articles. Our study is based on open-source data, so there are no ethical issues or conflicts of interest.

Footnotes: 
Publisher's Note

Springer Nature remains neutral with regard to jurisdictional claims in published maps and institutional affiliations.

Contributor Information:

Cangyuan Zhang, Email: zhangcy156707@foxmail.com.

Ziyang Long, Email: 1353212886@qq.com.

Cheng Yan, Email: 1182662102@qq.com.

Mohamed Said Jalloh, Email: 769783496@qq.com

Yongkun Fang, Email: 185857782@qq.com

Li Bao, Email: 2750347035@qq.com

Biao Sun, Email: 274958615@qq.com

Wenzhe Shao, Email: 420722831@qq.com

Guowei Sun, Email: 291644311@qq.com

Yayan Fu, Email: 1152016243@qq.com

Qi Zhang, Email: dx120190166@yzu.edu.cn.

Wei Wang, Email: wangwei815828@163.com

Liuhua Wang, Email: wlh1118ok@sina.com

Jun Ren, Email: freezingfall@163.com

Qiannan Sun, Email: 18042688505@163.com

Dong Tang, Email: 83392785@qq.com

Daorong Wang, Email: wdaorong666@sina.com.

\section{References}

1. Siegel RL, Miller KD, Fuchs HE, Jemal A: Cancer Statistics, 2021. CA: a cancer journal for clinicians 2021, 71(1):7-33. 
2. Nagasawa A, Kudoh J, Noda S, Mashima Y, Wright A, Oguchi Y, Shimizu N: Human and mouse ISLR (immunoglobulin superfamily containing leucine-rich repeat) genes: genomic structure and tissue expression. Genomics 1999, 61(1):37-43.

3. Maeda K, Enomoto A, Hara A, Asai N, Kobayashi T, Horinouchi A, Maruyama S, Ishikawa Y, Nishiyama T, Kiyoi H et al: Identification of Meflin as a Potential Marker for Mesenchymal Stromal Cells. Scientific reports 2016, 6:22288.

4. Zhang K, Zhang Y, Gu L, Lan M, Liu C, Wang M, Su Y, Ge M, Wang T, Yu Y et al: IsIr regulates canonical Wnt signaling-mediated skeletal muscle regeneration by stabilizing Dishevelled-2 and preventing autophagy. Nature communications 2018, 9(1):5129.

5. Hara A, Kobayashi H, Asai N, Saito S, Higuchi T, Kato K, Okumura T, Bando YK, Takefuji M, Mizutani Y et al: Roles of the Mesenchymal Stromal/Stem Cell Marker Meflin in Cardiac Tissue Repair and the Development of Diastolic Dysfunction. Circulation research 2019, 125(4):414-430.

6. Xu J, Tang Y, Sheng X, Tian Y, Deng M, Du S, Lv C, Li G, Pan Y, Song Y et al: Secreted stromal protein ISLR promotes intestinal regeneration by suppressing epithelial Hippo signaling. The EMBO journal 2020, 39(7):e103255.

7. Mizutani Y, Kobayashi H, lida T, Asai N, Masamune A, Hara A, Esaki N, Ushida K, Mii S, Shiraki Y et al: Meflin-Positive Cancer-Associated Fibroblasts Inhibit Pancreatic Carcinogenesis. Cancer research 2019, 79(20):5367-5381.

8. Martin JD, Miyazaki T, Cabral H: Remodeling tumor microenvironment with nanomedicines. Wiley interdisciplinary reviews Nanomedicine and nanobiotechnology 2021:e1730.

9. Ivey JW, Bonakdar M, Kanitkar A, Davalos RV, Verbridge SS: Improving cancer therapies by targeting the physical and chemical hallmarks of the tumor microenvironment. Cancer letters 2016, 380(1):330-339.

10. Bindea G, Mlecnik B, Tosolini M, Kirilovsky A, Waldner M, Obenauf AC, Angell H, Fredriksen T, Lafontaine L, Berger A et al: Spatiotemporal dynamics of intratumoral immune cells reveal the immune landscape in human cancer. Immunity 2013, 39(4):782-795.

11. De Palma M, Biziato D, Petrova TV: Microenvironmental regulation of tumour angiogenesis. Nature reviews Cancer 2017, 17(8):457-474.

12. Li X, Yao W, Yuan Y, Chen P, Li B, Li J, Chu R, Song H, Xie D, Jiang X et al: Targeting of tumourinfiltrating macrophages via CCL2/CCR2 signalling as a therapeutic strategy against hepatocellular carcinoma. Gut 2017, 66(1):157-167.

13. Monteran L, Erez N: The Dark Side of Fibroblasts: Cancer-Associated Fibroblasts as Mediators of Immunosuppression in the Tumor Microenvironment. Frontiers in immunology 2019, 10:1835.

14. Wang Z, Yang Q, Tan Y, Tang Y, Ye J, Yuan B, Yu W: Cancer-Associated Fibroblasts Suppress Cancer Development: The Other Side of the Coin. Frontiers in cell and developmental biology 2021, 9:613534.

15. Rhodes DR, Kalyana-Sundaram S, Mahavisno V, Varambally R, Yu J, Briggs BB, Barrette TR, Anstet MJ, Kincead-Beal C, Kulkarni P et al: Oncomine 3.0: genes, pathways, and networks in a collection of 
18,000 cancer gene expression profiles. Neoplasia (New York, NY) 2007, 9(2):166-180.

16. Goldman MJ, Craft B, Hastie M, Repečka K, McDade F, Kamath A, Banerjee A, Luo Y, Rogers D, Brooks AN et al: Visualizing and interpreting cancer genomics data via the Xena platform. Nature biotechnology 2020, 38(6):675-678.

17. Nagy Á, Munkácsy G, Győrffy B: Pancancer survival analysis of cancer hallmark genes. Scientific reports 2021, 11(1):6047.

18. Li T, Fan J, Wang B, Traugh N, Chen Q, Liu JS, Li B, Liu XS: TIMER: A Web Server for Comprehensive Analysis of Tumor-Infiltrating Immune Cells. Cancer research 2017, 77(21):e108-e110.

19. Ru B, Wong CN, Tong Y, Zhong JY, Zhong SSW, Wu WC, Chu KC, Wong CY, Lau CY, Chen I et al: TISIDB: an integrated repository portal for tumor-immune system interactions. Bioinformatics (Oxford, England) 2019, 35(20):4200-4202.

20. Yu G, Wang LG, Han Y, He QY: clusterProfiler: an R package for comparing biological themes among gene clusters. Omics : a journal of integrative biology 2012, 16(5):284-287.

21. Chang L, Chang M, Chang HM, Chang F: Microsatellite Instability: A Predictive Biomarker for Cancer Immunotherapy. Applied immunohistochemistry \& molecular morphology : AlMM 2018, 26(2):e15e21.

22. Hellmann MD, Ciuleanu TE, Pluzanski A, Lee JS, Otterson GA, Audigier-Valette C, Minenza E, Linardou $\mathrm{H}$, Burgers S, Salman $\mathrm{P}$ et al: Nivolumab plus Ipilimumab in Lung Cancer with a High Tumor Mutational Burden. The New England journal of medicine 2018, 378(22):2093-2104.

23. Kobayashi H, Gieniec KA, Wright JA, Wang T, Asai N, Mizutani Y, Lida T, Ando R, Suzuki N, Lannagan TRM et al: The Balance of Stromal BMP Signaling Mediated by GREM1 and ISLR Drives Colorectal Carcinogenesis. Gastroenterology 2021, 160(4):1224-1239.e1230.

24. Ohara Y, Enomoto A, Tsuyuki Y, Sato K, lida T, Kobayashi H, Mizutani Y, Miyai Y, Hara A, Mii S et al: Connective tissue growth factor produced by cancer-associated fibroblasts correlates with poor prognosis in epithelioid malignant pleural mesothelioma. Oncology reports 2020, 44(3):838-848.

25. Malenica I, Adam J, Corgnac S, Mezquita L, Auclin E, Damei I, Grynszpan L, Gros G, de Montpréville V, Planchard D et al: Integrin-a(V)-mediated activation of TGF- $\beta$ regulates anti-tumour CD8 T cell immunity and response to PD-1 blockade. Nature communications 2021, 12(1):5209.

26. Yang Z, Xu G, Wang B, Liu Y, Zhang L, Jing T, Tang M, Xu X, Jiao K, Xiang L et al: USP12 downregulation orchestrates a protumourigenic microenvironment and enhances lung tumour resistance to PD-1 blockade. Nature communications 2021, 12(1):4852.

27. Bejarano L, Jordāo MJC, Joyce JA: Therapeutic Targeting of the Tumor Microenvironment. Cancer discovery 2021, 11(4):933-959.

28. Snyder A, Makarov V, Merghoub T, Yuan J, Zaretsky JM, Desrichard A, Walsh LA, Postow MA, Wong P, Ho TS et al: Genetic basis for clinical response to CTLA-4 blockade in melanoma. The New England journal of medicine 2014, 371(23):2189-2199.

29. Le DT, Uram JN, Wang H, Bartlett BR, Kemberling H, Eyring AD, Skora AD, Luber BS, Azad NS, Laheru D et al: PD-1 Blockade in Tumors with Mismatch-Repair Deficiency. The New England journal of 
medicine 2015, 372(26):2509-2520.

30. Cristescu R, Mogg R, Ayers M, Albright A, Murphy E, Yearley J, Sher X, Liu XQ, Lu H, Nebozhyn M et al: Pan-tumor genomic biomarkers for PD-1 checkpoint blockade-based immunotherapy. Science (New York, NY) 2018, 362(6411).

31. Lin A, Zhang J, Luo P: Crosstalk Between the MSI Status and Tumor Microenvironment in Colorectal Cancer. Frontiers in immunology 2020, 11:2039.

\section{Figures}


(A)

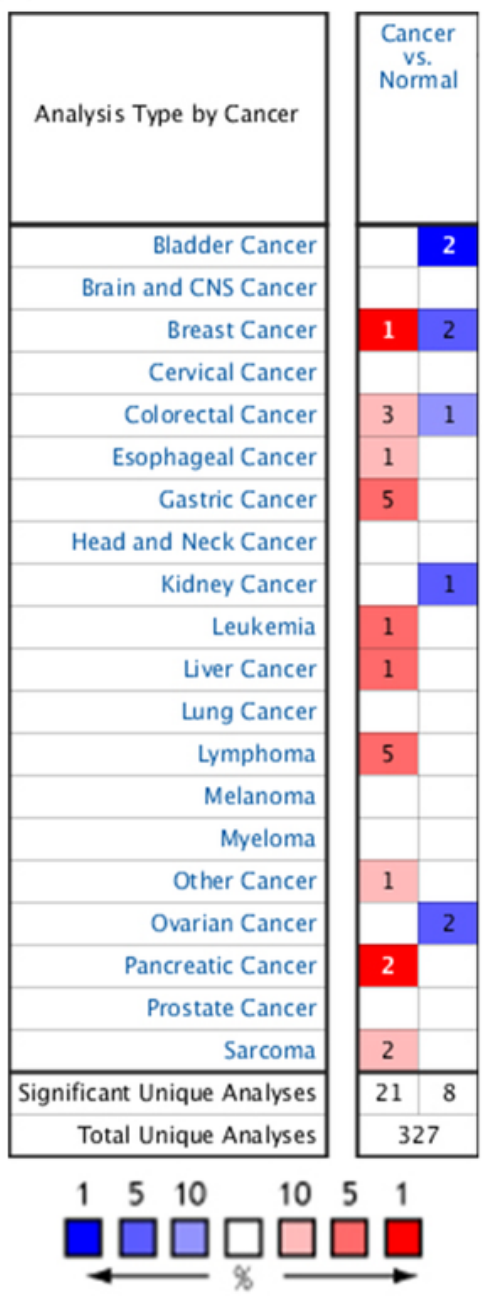

Cell color is determined by the best gene rank percentile for the analyses within the cell.

(E)
(B) Gender 白 temale 白 mate

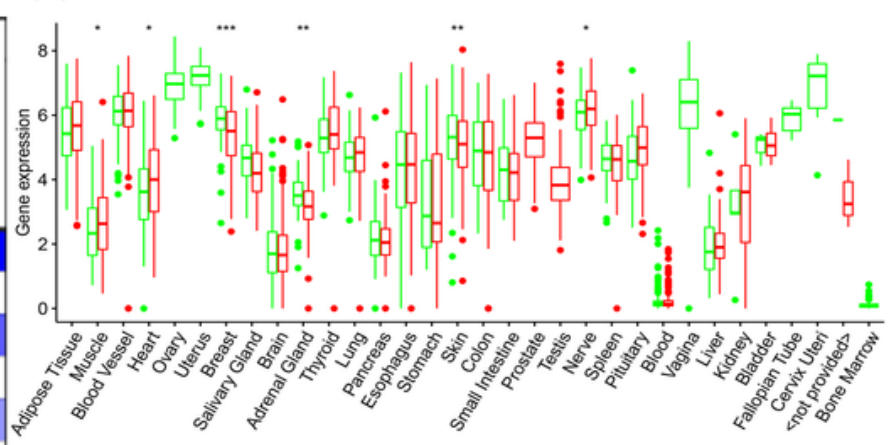

(C)
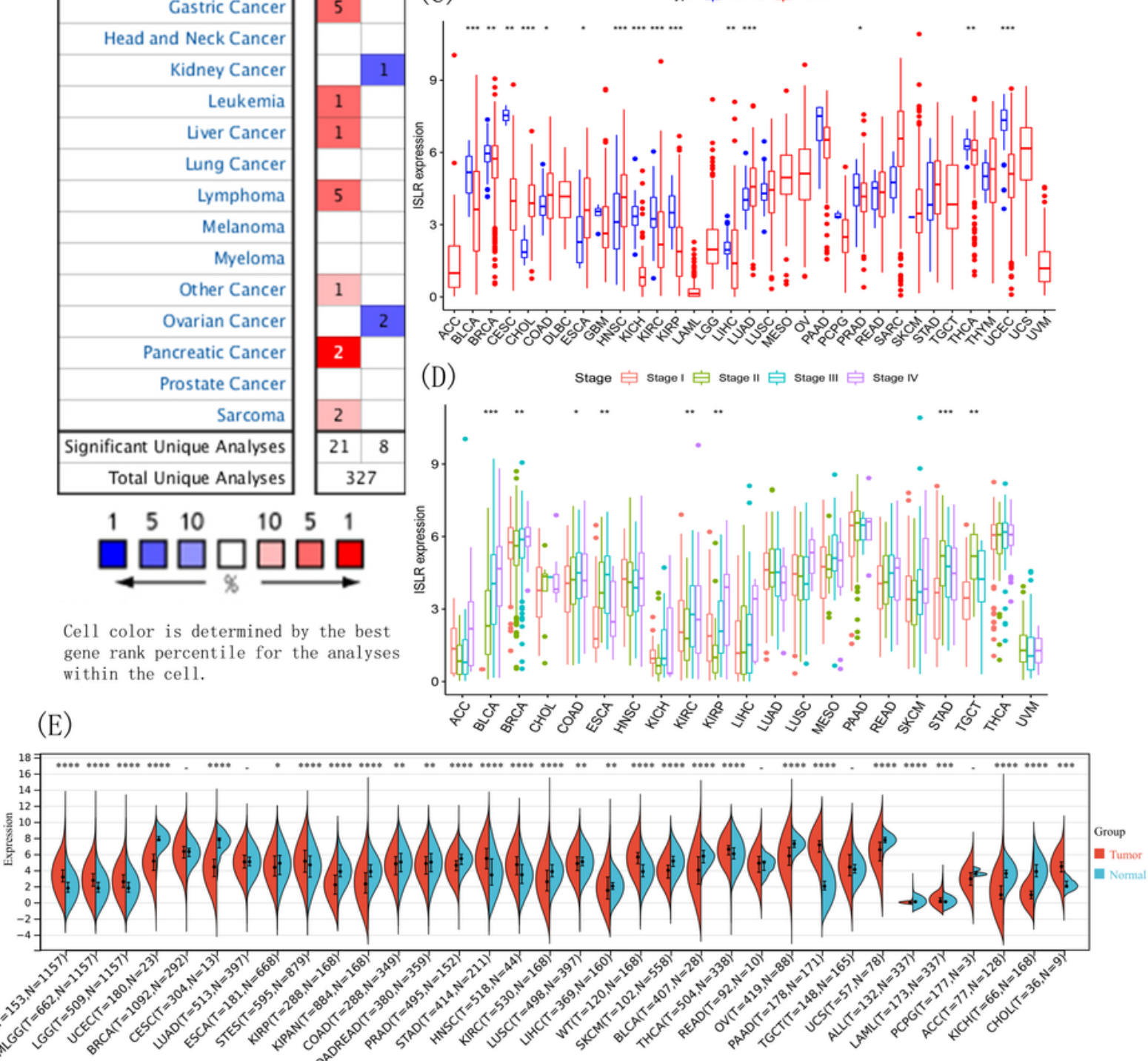
Sangerbox online tool analyzes the differential expression of ISLR in each tumor under the combined data of TCGA and GTEx. All statistical data followed ${ }^{\star} \mathrm{P}<0.05$, ${ }^{\star *} \mathrm{P}<0.01, \star \star \star \mathrm{P}<0.001,{ }^{\star \star \star \star *} \mathrm{P}<0.0001$.
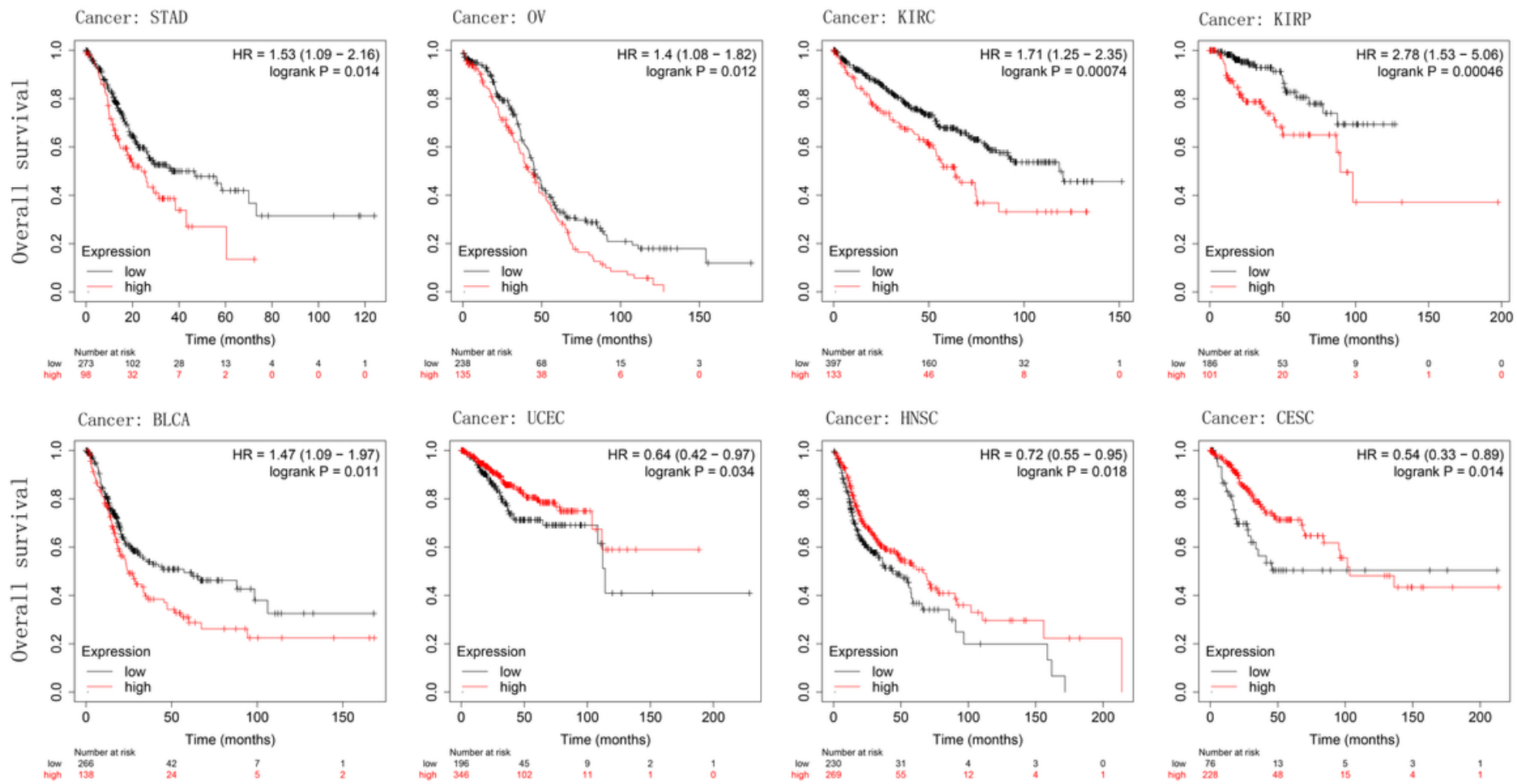

\section{Figure 2}

Kaplan-Meier analyzed the relationship between ISLR expression and OS. Red represents the ISLR high expression group, black represents the ISLR low expression group. The horizontal axis represents survival time, and the vertical axis represents overall survival. $H R>0$ means a positive correlation, while $H R<0$ means a negative correlation. 

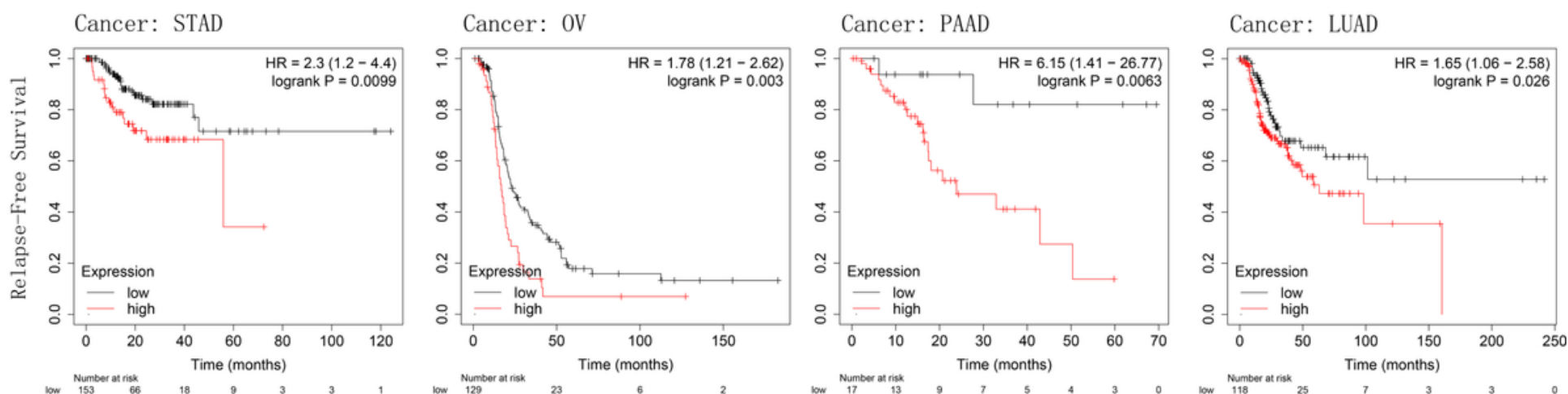

Cancer: TGCT

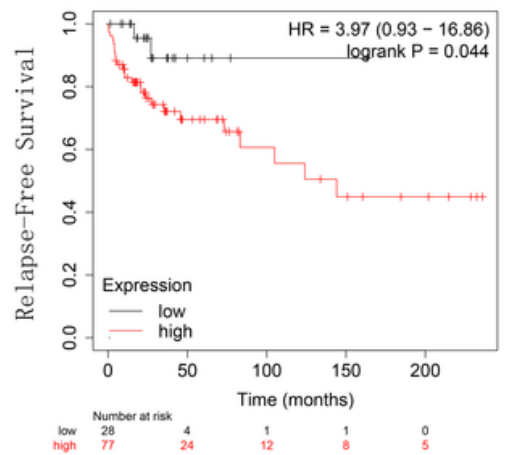

Cancer: Esophageal Squamous Cell Carcinoma

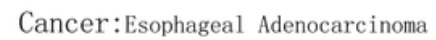

Cancer: HNSC
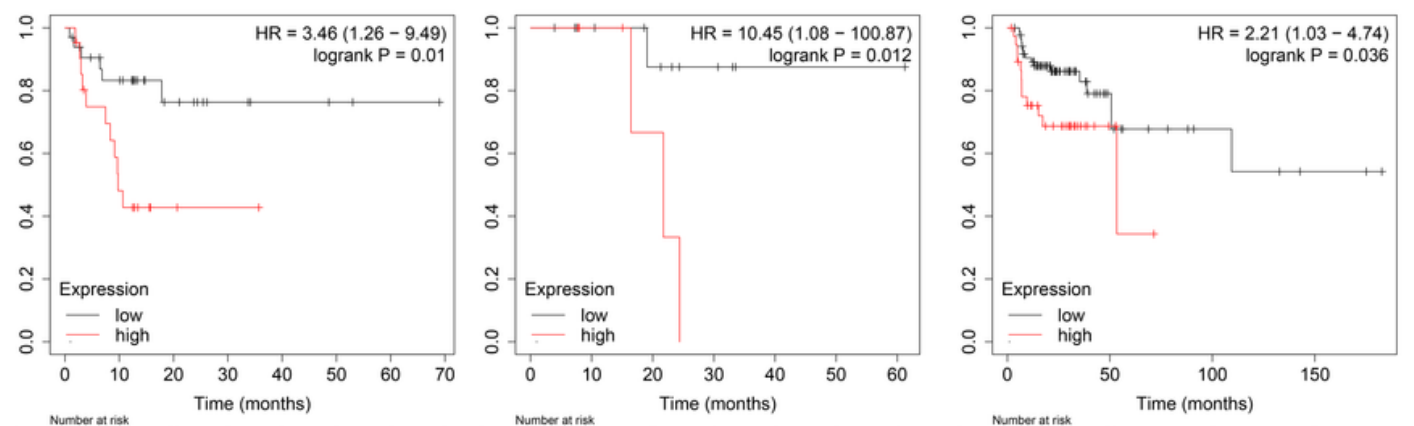

Cancer: LIHC

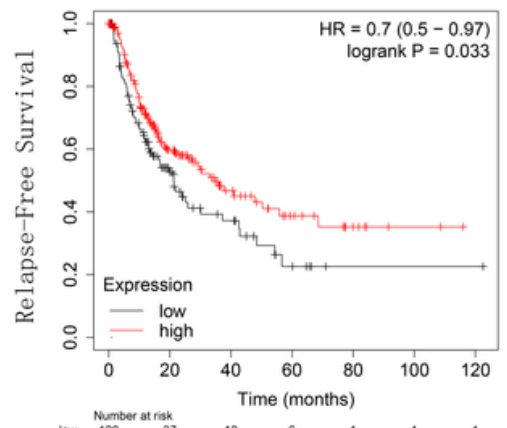

Cancer: THCA

Cancer: UCEC
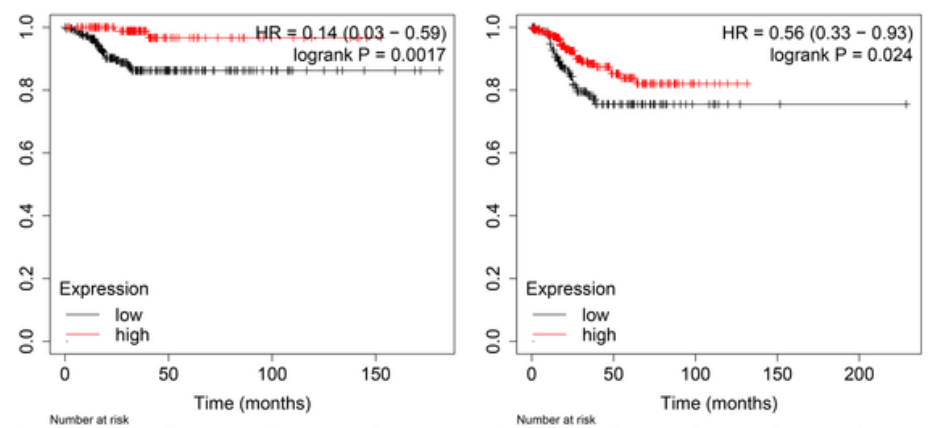

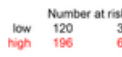

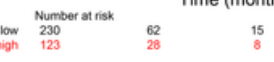

low
lion
high
263

Figure 3

Kaplan-Meier analyzed the relationship between ISLR expression and RFS. Red represents the group with high ISLR expression and black represents the group with low ISLR expression. The horizontal axis is survival time, and the vertical axis is RFS. HR $>0$ means a positive correlation, while $H R<0$ means a negative correlation. 
(A) Cancer: ACC

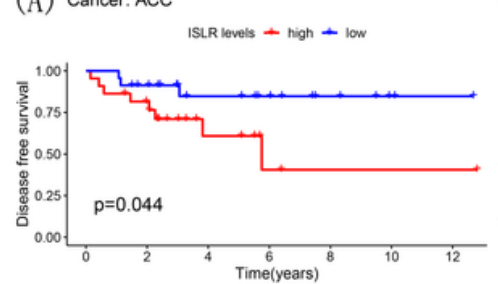

(B) Cancer: ESCA

ISLR lovels + high + low

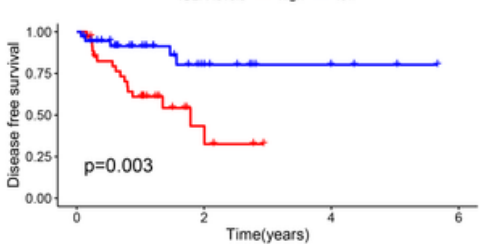

(C) Cancer: PAAD

18 R

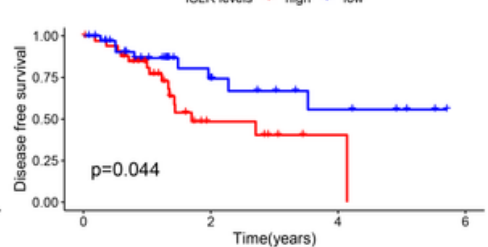

(D) Cancer: THCA

ISLR levels + nigh + Iow
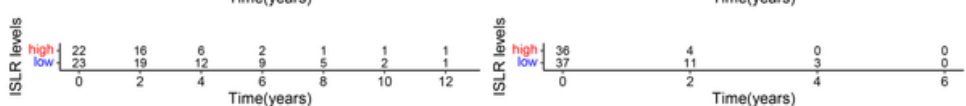

(F) Cancer: KIRC

(E) Cancer: UCEC

ISLR levels + high + low
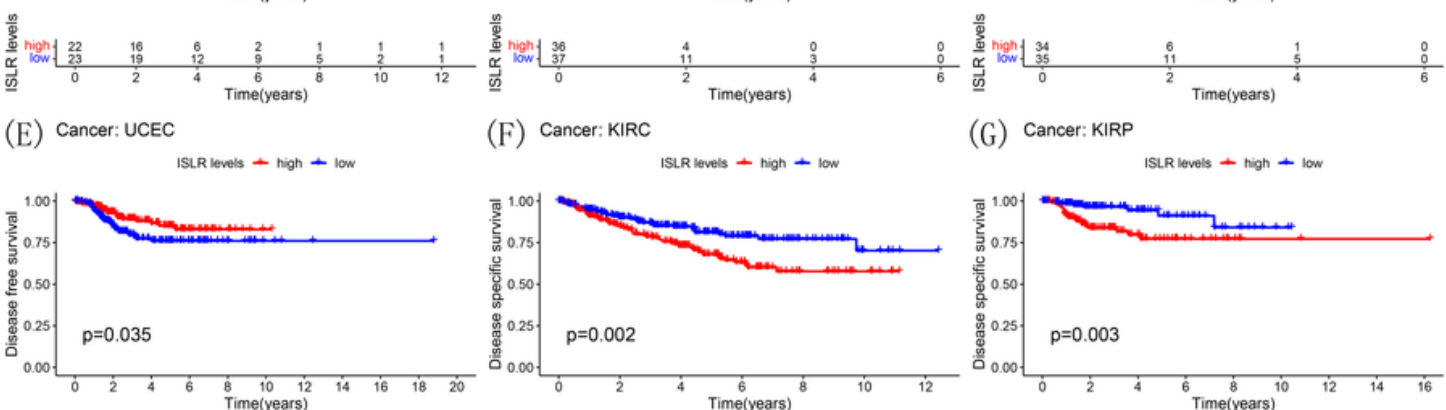

(G) Cancer: KIRP
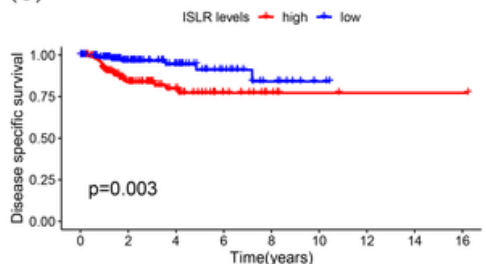

Cancer: STAD

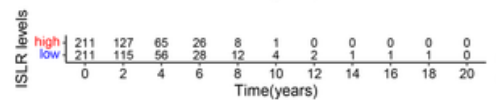

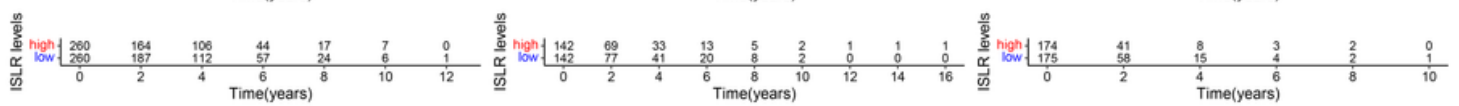

(I) Cancer: KIRC
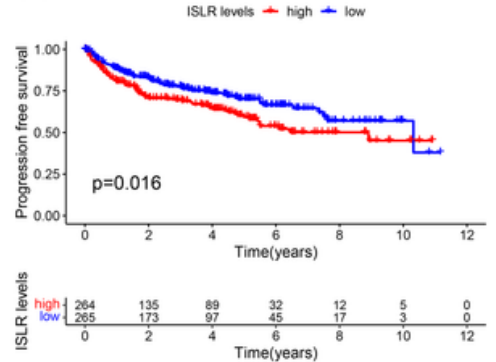

\section{Figure 4}

(A)- (E) is the relationship between ISLR expression and DFS such as ACC, ESCA, PAAD, THCA and UCEC in turn. $(F)-(H)$ in turn is the relationship between ISLR expression and DSS such as KIRC, KIRP, STAD. (I) is the relationship between ISLR expression and KIRC's PFS. Red represents the ISLR high expression group, and blue represents the ISLR low expression group. 

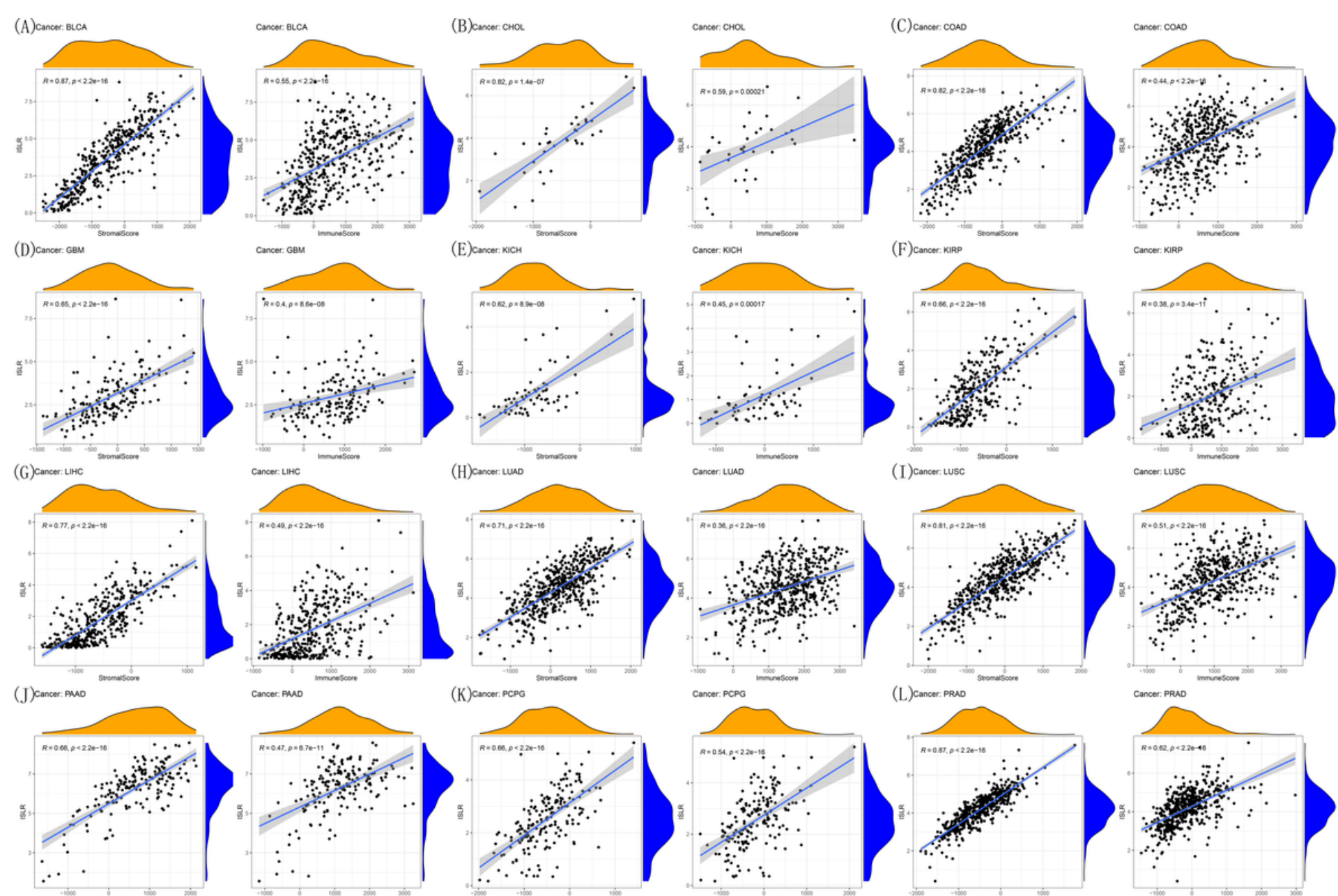

$(\omega \rightarrow \infty)$
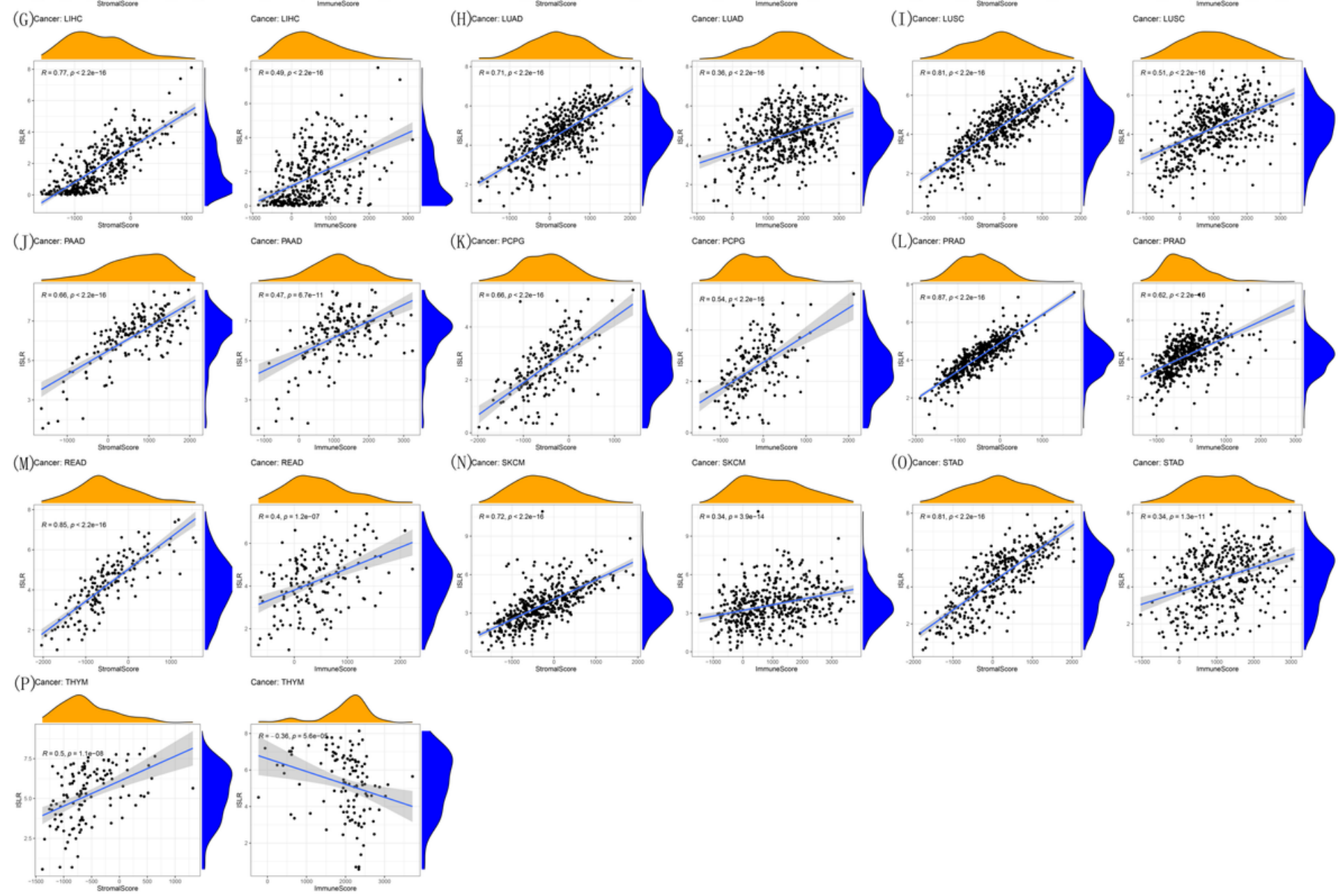

\section{Figure 5}

(A)- $(\mathrm{P})$ are the correlation analysis between the tumor stromal scores and immune scores of ISLR expression part. The $P$ values were all $<0.001$, and the correlation coefficient was $>0.3$. 

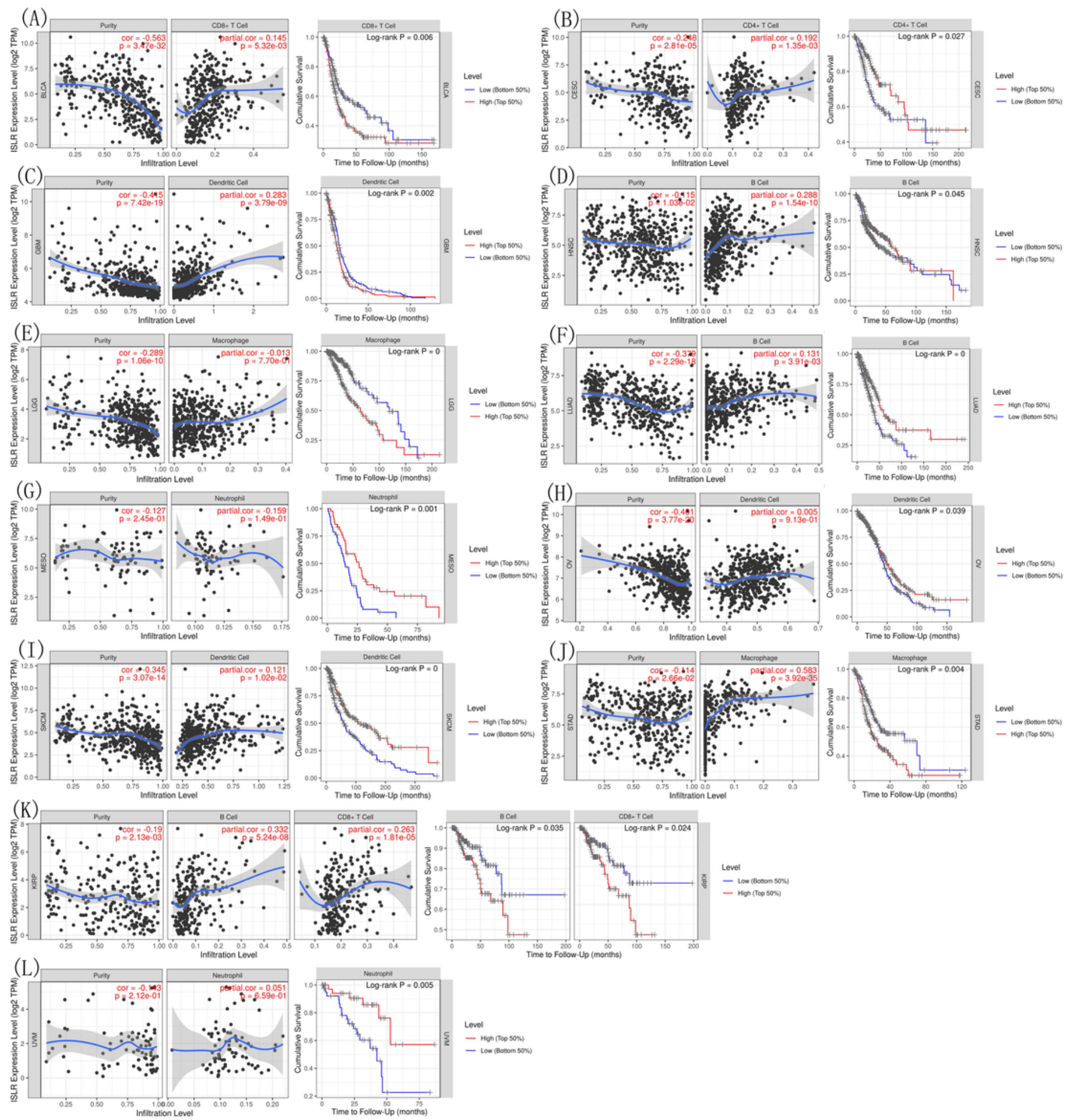

\section{Figure 6}

The amount of immune cell infiltration affects the clinical prognosis and is correlated with the expression of ISLR. Cor $>0$ means positive correlation. Cor<0 means negative correlation. 


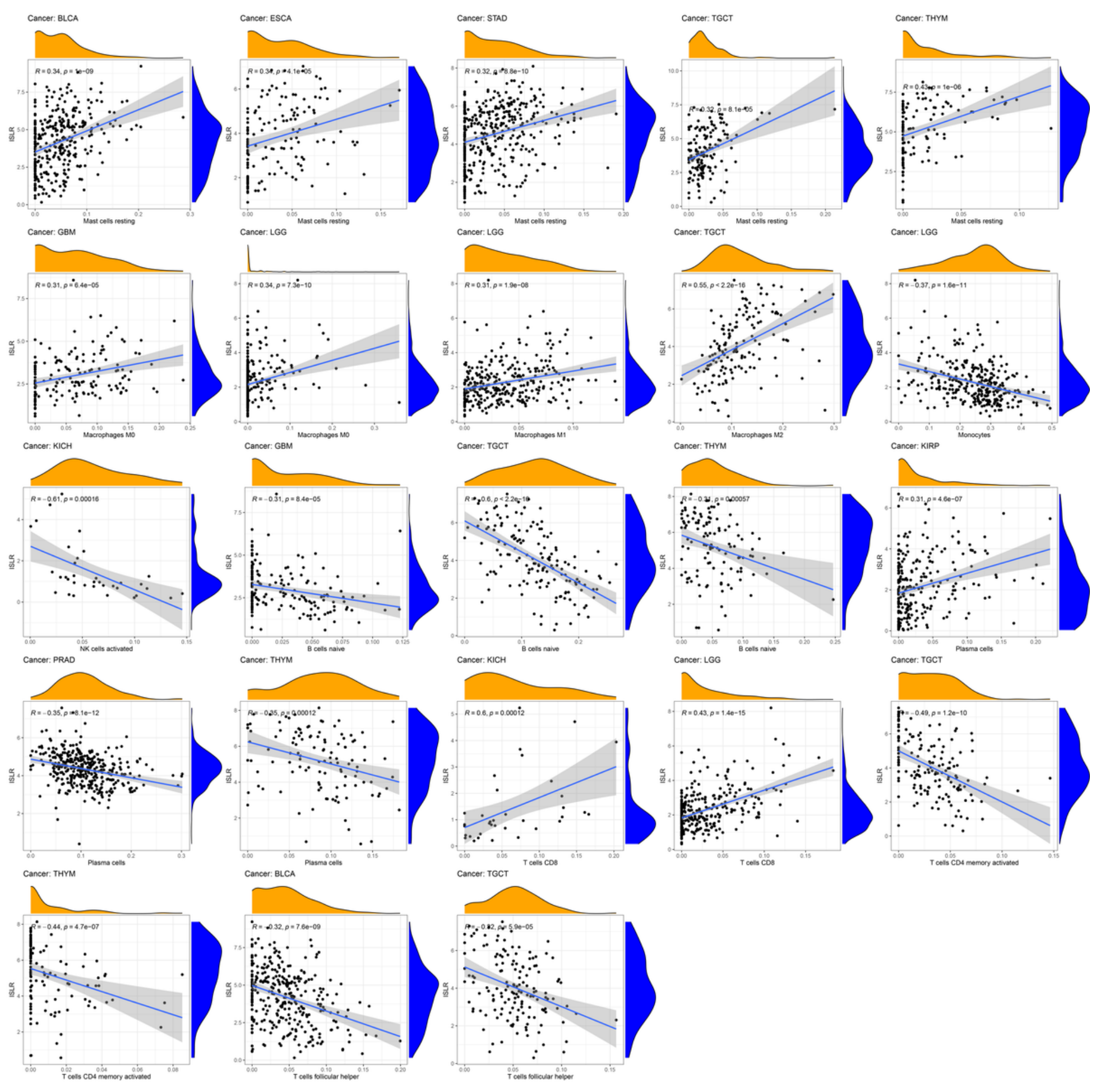

Figure 7

The expression of ISLR in pan-cancer affects the level of infiltration of different immune cell subtypes. 

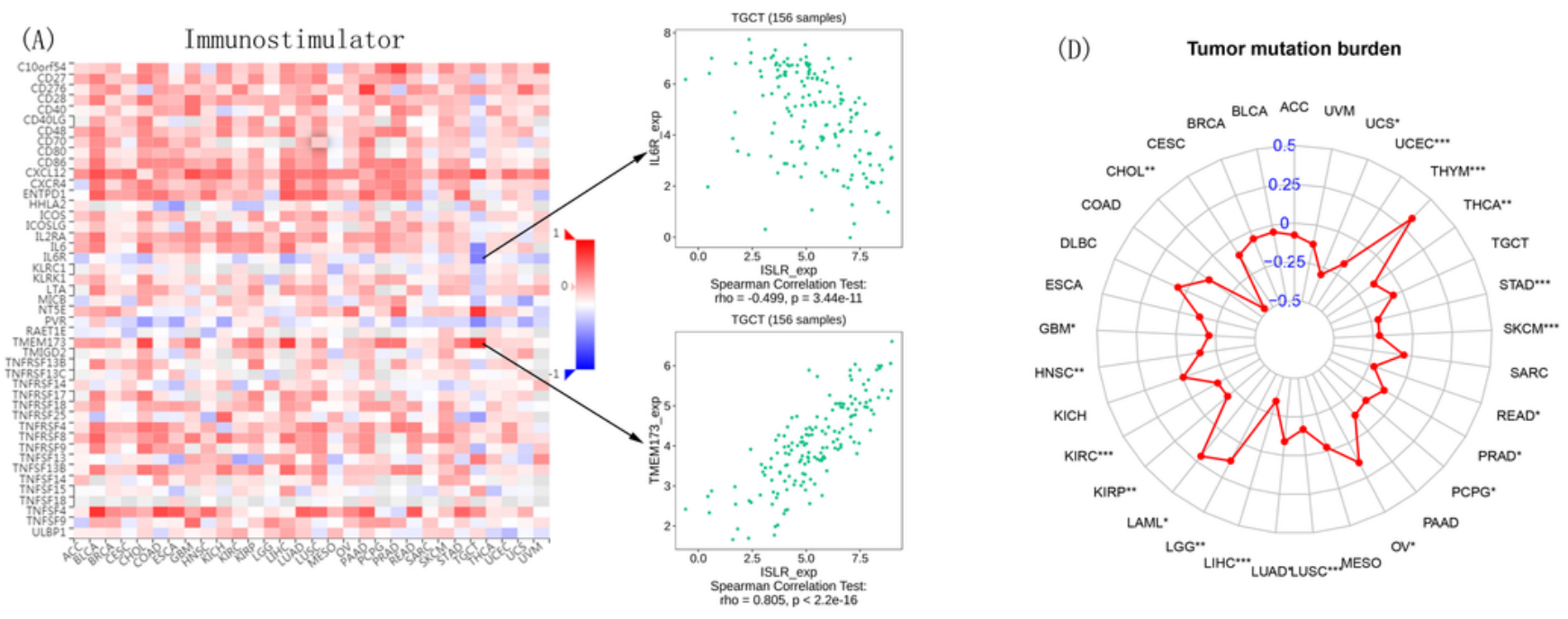

(B) Immunoinhibitor
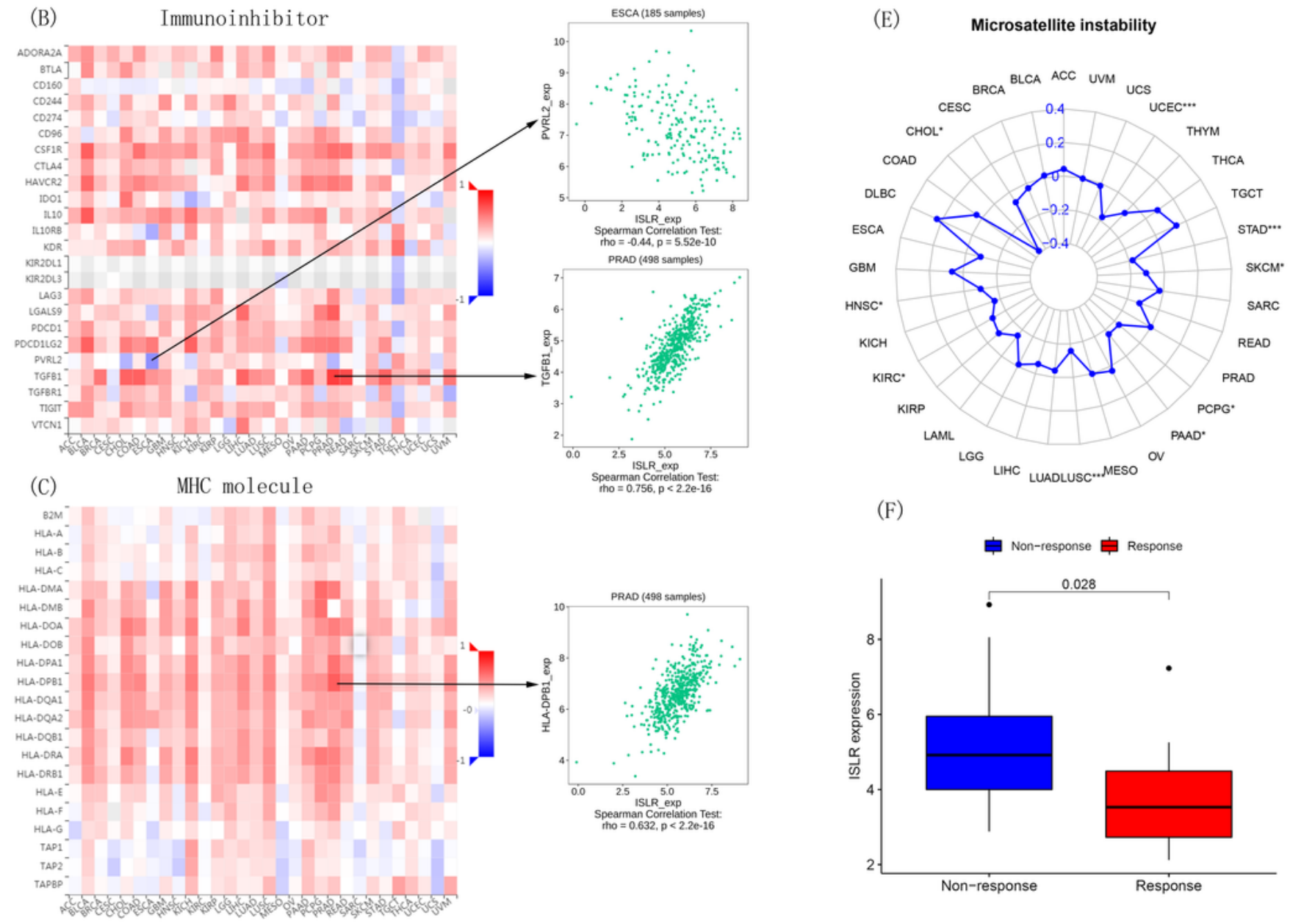

\section{Figure 8}

(A) ISLR expression is correlated with immunostimulatory genes. (B) ISLR expression regulates immunosuppressive gene expression. (C) ISLR expression regulates MHC-related gene expression. The abscissa represents tumor type, the ordinate represents immune genes, red represents positive correlation, blue represents negative correlation, and white represents no correlation. The right side of (A)(C) is a box plot between the most correlated immune gene and ISLR expression. (D) The radar chart 
shows the correlation between ISLR expression and tumor mutational burden (TMB) in all cancer types. The correlation coefficient is indicated by a red line, and the range is indicated by a blue line. (E) Radar chart showing the correlation between ISLR expression and microsatellite instability (MSI) in all cancer types. The correlation coefficient is represented by the blue line, and the mesh line represents the range. (F) The relationship between ISLR expression in the GSE78220 data set and the effect of melanoma immunotherapy. Blue represents the immunotherapy non-response group, and red represents the immunotherapy response group.
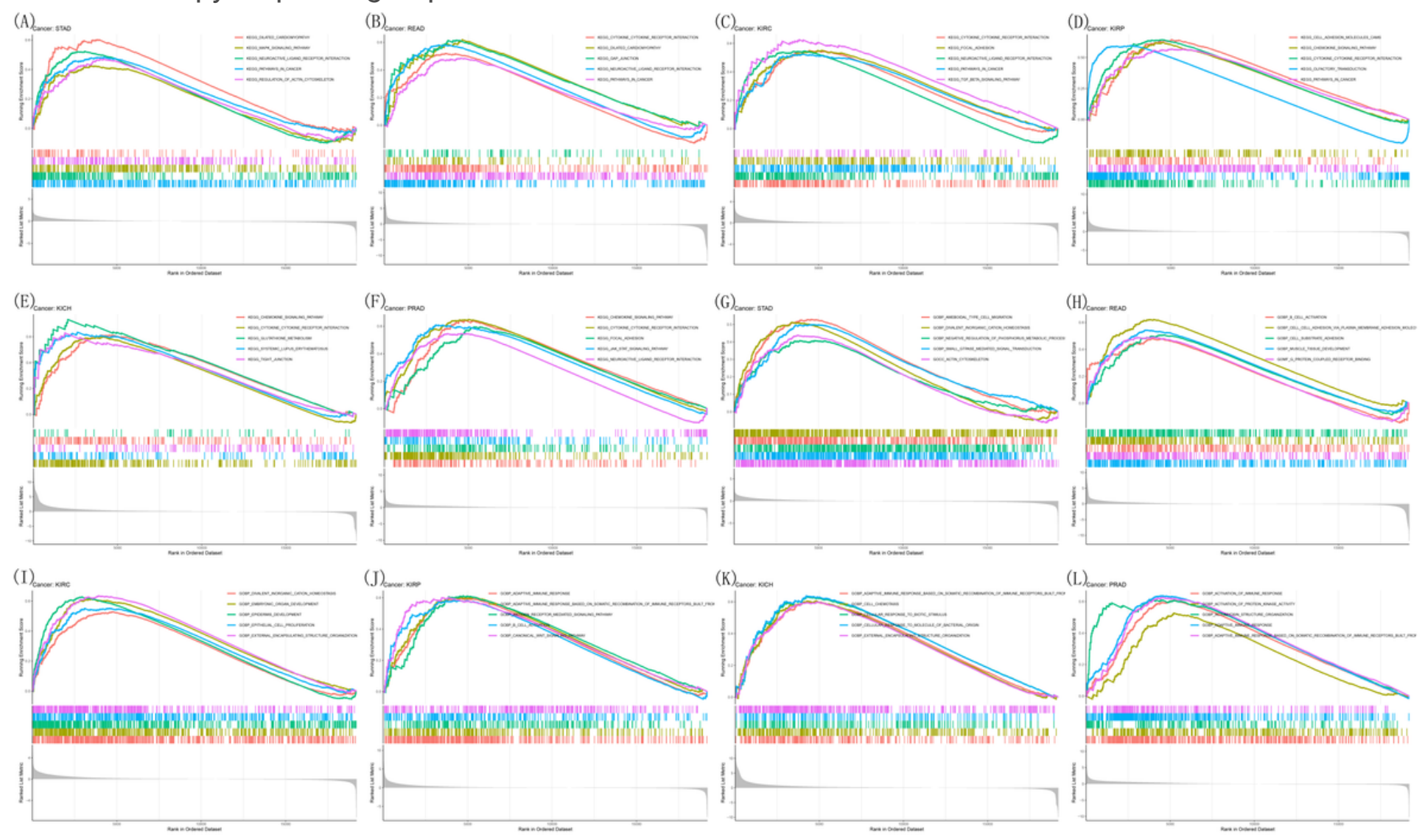

\section{Figure 9}

(A)- (F) are the KEGG pathway analysis of ISLR in STAD, READ, KIRC, KIRP, KICH, and PRAD respectively. (G)- (L) are the GO function annotations of ISLR in STAD, READ, KIRC, KIRP, KICH, PRAD. The different functions and pathways of ISLR in various tumors are represented by curves with different colors. An upward curve peak means positive regulation, and a downward curve peak means negative regulation.

\section{Supplementary Files}

This is a list of supplementary files associated with this preprint. Click to download.

- SupplementaryFigure2.pdf

- SupplementaryFigureDFS.pdf

- SupplementaryFigureDSS.pdf 
- SupplementaryFigureLegends.docx

- SupplementaryFigurePFS.pdf

- Supplementarymaterial.rar

- SupplementaryTable1a.docx

- SupplementaryTable1b.xlsx

- SupplementaryTable1c.xlsx 